\title{
The lift pool method for isolation of cDNA clones from lambda phage libraries
}

\author{
Janine LeBlanc-Straceski* \\ Department of Biology and Allied Health \\ Merrimack College \\ North Andover Massachusetts 01845 \\ Te: $9788375000 / 4357$ \\ Fax: 9788375180 \\ E-mail: Janine.LeBlancStraceski@merrimack.edu
}

Pablo Sobrado

University of Wisconsin, Madison

Biochemistry Department

433 Babcock Drive Room 123

Madison, WI 53706

Tel: 6082623364 Ext 2110

E-mail: psobrado@biochem.wisc.edu

\section{Sharon Betz}

Clinical Molecular Genetics Fellow

McLendon Clinical Labs

Department of Pathology and Laboratory Medicine

University of North Carolina CB \#7525

Chapel Hill, NC 27599

Tel: 9199664408

Fax: 9199666351

E-mail:sbetz@unch.unc.edu

\section{Julie Zerfas}

Building K.

Wyeth BioPharma

1 Burtt Rd., Andover, MA 01810

Tel: 9782474295

Fax: 9782474298

E-mail: jzerfas@wyeth.com

\section{Karen Morgan}

College of Osteopathic Medicine

University of New England

11 Hills Beach Road

Biddeford, ME 04005-9599

E-mail: kmorgan1@pipeline.une.edu

Financial support: The Faculty Development Grant Program of Merrimack College, Merck/AAAS Undergraduate Science Research Program grant, a National Science Foundation-Undergraduate Research Institution grant (NSF-URI) NSF0077516.

Keywords: cDNA, lambda, non-radioactive, PCR, plaque, screening.

Abbreviations: LP: lift pool

PCR: polymerase chain reaction

Pfu: plaque forming unit

PP: plate pool

SP: super poo

XIMyo1d: Xenopus laevis myosin 1d

XIMyo7a: Xenopus laevis myosin 7a

A PCR based strategy was developed to screen a Xenopus oocyte Agt10 cDNA library. The PCR-based lift pool (LP) method follows the same two tiered strategy as conventional screening of phage libraries by

* Corresponding author 
filter hybridization. Two rounds of plating, one at high density to detect the clone, and one at low density to purify the clone to homogeneity, are performed. In the first round, lysates from high density plates, termed plate pools (PP), serve as template for PCR. In the second round, phage particles adhering to plaque lifts of low density plates are washed off nitrocellulose filters to create LPs, which are used as template for PCR. The integrity of the plaques on the low-density plates is preserved. Once a positive LP has been identified, plaques on the corresponding plate are screened individually by PCR. Using isoform specific primer pairs for Xenopus myosin $7 \mathrm{a}$ and myosin 1d, two lambda clones were isolated. Subsequent DNA sequence analysis confirmed their identities as myosin isoforms (GenBank accession numbers: DQ100353 and AF540952). This method offers a time saving, costeffective alternative to other hierarchical pooling strategies for the repeated screening by PCR of an arrayed lambda phage library.

PCR based screening strategies for lambda libraries have been developed as efficient, non-radioactive alternatives to colorimetric or chemiluminescent detection of nucleic acid probes hybridized to plaque lifts (Rosenberg et al. 1991; Dorfman, 1993; Gonzalez and Chan, 1993; Amaravadi and King, 1994; D'Esposito et al. 1994; McAlinden and Krawetz, 1994; Munroe et al. 1995; Yu and Bloem, 1996; Alphey, 1997; Takumi, 1997; Watanabe, 1997). Conventional screening of $\lambda$ phage libraries by filter hybridization involves two rounds of plating: one at high density to detect the clone, and one at low density to purify the clone to homogeneity. Many PCR based methods follow this two-tiered strategy invoking similar pooling strategies for initial detection, but different steps to isolate the final purified $\lambda$ clones of interest. Here we describe a streamlined, robust alternative to the second tier of screening. In the first round, lysates from high density plates, termed plate pools (PPs), serve as template for PCR (Figure 1, steps 1-6). Then, single PPs are re-plated at low density (Figure 1, step 7). Phage particles adhering to plaque lifts of these low density plates are washed off nitrocellulose filters to create lift pools (LPs), which are used as template for PCR (Figure 1, steps 8-9). The integrity of the plaques on the plates is preserved. Once a positive LP has been identified, plaques on the corresponding plate are screened individually, again by PCR (Figure 1, step 10).
1. Plate lambda phage library on $100 \mathrm{~mm}$ plates at high density

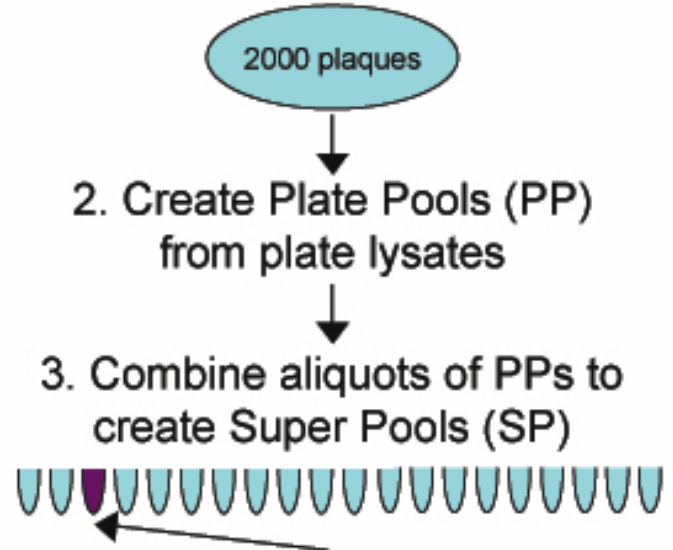

4. Identify a positive SP by PCR

5. Screen the PPs* by PCR

("whose aliquots were combined to create the SP)

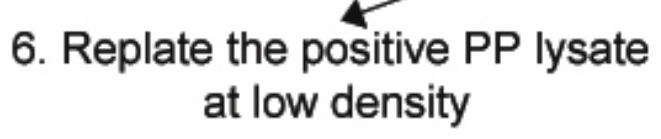

\section{Place filters on low density plates}
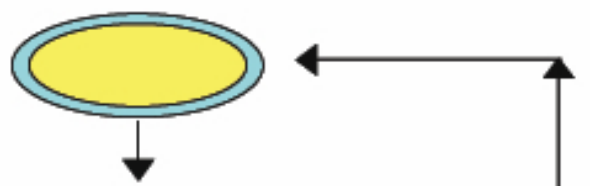

8. Remove filters and wash off adhering particles to create Lift Pools (LP)

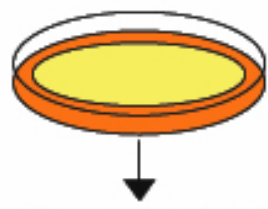

9. Screen LPs via PCR to identify positive low density plate 00000000000000000000 00000000000000000

10. Go back to original low density plate and screen individual plaques to identify the lambda clone

Figure 1. The LP screening method. The strategy is based on PCR detection of $\lambda$ phage cDNA clones in pools created from high and low density plates. The integrity of low density plates in step 7 is preserved because phage are not eluted directly from the agar, neither by flooding the plate, nor by elution of phage from slices of agar from the plate. Instead plaques adhering to a nitrocellulose filter placed on the plate are washed off and used as template for the PCR screen. Once a positive low density plate is identified, individual phage eluted from agar plugs can be screened to identify the clone. Details of the protocol are described in the Materials and Methods. 


\section{MATERIALS AND METHODS}

Primers for PCR were derived from a 93 bp gene specific sequence of the Xenopus laevis myosin 7a (XIMyo7a) homologue, and a $102 \mathrm{bp}$ sequence of XIMyo1d (Sokac and Bement, 2000).

Forward, [CACCAAACTTCTGCTCAAGC], and reverse, [CAATAAGGGATTTACCTGTAGGA], primers were used to generate an $88 \mathrm{bp}$ fragment specific to the XIMyo $7 \mathrm{a}$ isoform. For the XIMyold isoform, the forward primer [ATGGGCTACATCTCCAAGGT] and reverse primer [GGGGGTTGGATTTGAGAATA] were used to generate a 79 bp fragment. Each $25 \mu$ PCR reaction contained 2.5 $\mu 1,10 \mathrm{X}$ Sigma Taq polymerase buffer; $2 \mu 1,2.5 \mathrm{mM}$ dNTPs; $2 \mu \mathrm{l}, 25 \mathrm{mM} \mathrm{MgCl} 2 ; 0.2 \mu \mathrm{l}$ (1 unit) of Taq polymerase; $1 \mu \mathrm{l}$ each of $50 \mathrm{pmol} / \mu 1$ forward and reverse primers; 2 to $5 \mu \mathrm{l}$ of phage suspension and $\mathrm{H}_{2} \mathrm{O}$ to $25 \mu \mathrm{l}$. Reactions were carried out as follows: 30 cycles of $94^{\circ} \mathrm{C}$, $60^{\circ} \mathrm{C}$ and $72^{\circ} \mathrm{C}$ for 30 seconds each. All PCR products were electrophoresed through 3\% agarose gels in TBE buffer (Sambrook et al. 1989).
BNN102 cells were infected by a $\lambda$ gt10 library (Rebagliati et al. 1985) and were plated on NZCYM agar (Sambrook et al. 1989). After the initial titre was established, 400,000 pfu of the library was plated at a concentration of 2000 pfu per $100 \mathrm{~mm}$ plate. 200 plate lysates were prepared according to conventional methods by flooding the plates with SM buffer (Sambrook et al. 1989). Live phage were harvested, treated with $\mathrm{CHCl}_{3}$ and centrifuged to remove cellular debris (Sambrook et al. 1989). These cleared lystes or PPs were stored at $4^{\circ} \mathrm{C}$. Twenty super pools (SPs) were created by pooling $10 \mu \mathrm{l}$ aliquots of ten PPs. For example, aliquots of PPs 1-10 were combined to create SP1. Phage suspensions were used directly as the source of template DNA for PCR without further purification.

LPs were created from single positively identified PPs. The stored PP lysate was plated at a density of 100 pfu onto forty plates for a total of $4000 \mathrm{pfu}$. This number is twice the actual number of pfu used to inoculate the PP in order to build redundancy into the screen. After plaques were formed, sterile BA85 (Schleicher and Shuell) nitrocellulose

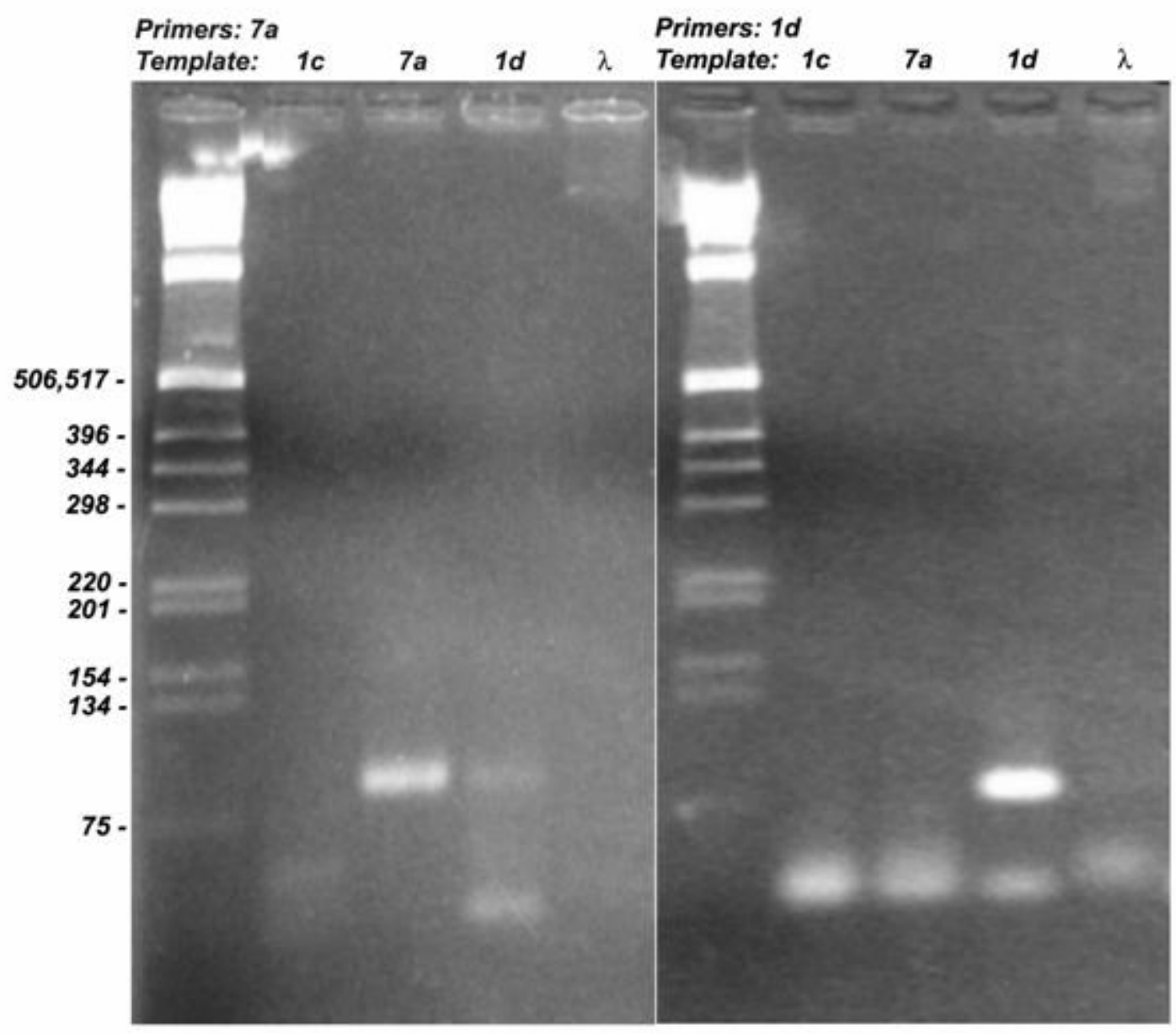

Figure 2. cDNA Specific Primer Pairs for PCR Screen of $\lambda$ gt10 Library. Primer pairs were designed to identify two different cytoplasmic myosin isoforms XIMyo7a and XIMyo1d from short cloned regions in an isoform specific region of the myosin head region. Primer pairs were tested against four templates, XIMyo1c, 7a and 1d, and lambda DNA, using the reaction conditions described in the Materials and Methods. The panel on the left shows products amplified using the $7 a$ primer pair. The panel on the right shows similar results for $1 \mathrm{~d}$ primer pair. 
filters were placed on top of the low density plates for 3 $\min$. The filters were carefully removed and placed into a sterile Petri dish containing $3 \mathrm{ml} \mathrm{SM}$ buffer on a rocking platform for $5 \mathrm{~min}$. The SM buffer containing phage was carefully removed into a sterile tube, shaken with a few drops of $\mathrm{CHCl}_{3}$ and centrifuged to remove cellular debris. The supernatant or LP was used directly as a source of template DNA for screening by PCR. Finally, plugs containing isolated plaques were removed, vortexed with $100 \mu 1$ of SM buffer, and centrifuged for $5 \mathrm{~min}$ at maximum RPM in a micro-centrifuge to remove agarose and cellular debris. Supernatants were screened by PCR using 2-5 $\mu \mathrm{l}$ of the plaque suspensions.

\section{RESULTS}

The LP method (Figure 1) was devised to isolate sequences homologous to two unconventional myosin heavy chain from a Xenopus oocyte $\lambda$ gt10 cDNA library (Rebagliati et al. 1985). Myosin heavy chains constitute a large, multigene family with many classes each containing several isoforms. However, isoform specific sequences exist within all myosin (Sokac and Bement, 2000). Isoform specific primer pairs that amplify an 88 bp region from Xlmyo7a and a 79 bp region of XlMyold were used in all screening steps (Figure 2). Before plating the cDNA library, an aliquot was used as template for PCR reactions using the isoform specific primers. Both sets of primers amplified the predicted products (data not shown).

The results of the first round of PCR screening in which the SPs were used as templates are shown in Figure 3. The XIMyo7a screen identified two positive SPs and four SPs were identified in the XlMyold screen. The ten PPs each from SP10 (for myosin 7a) and SP6 (for myoisn 1d) were screened in the second round of PCR. Each identified a single PP (data not shown) that was subsequently used to create sets of 40 LPs (Figure 1, steps 7 and 8).

Figure 4 shows the products of the myosin 7 a screen of a set of LPs. The 88 bp product was detected in LP 7 and LP 24. Once the Xlmyo7a positive LPs were identified, plaques from the original low density plates were screened individually (Figure 1, step 10). Subsequent DNA sequence analysis of the individual lambda clones purified from this screen confirmed that they contain the sequence from

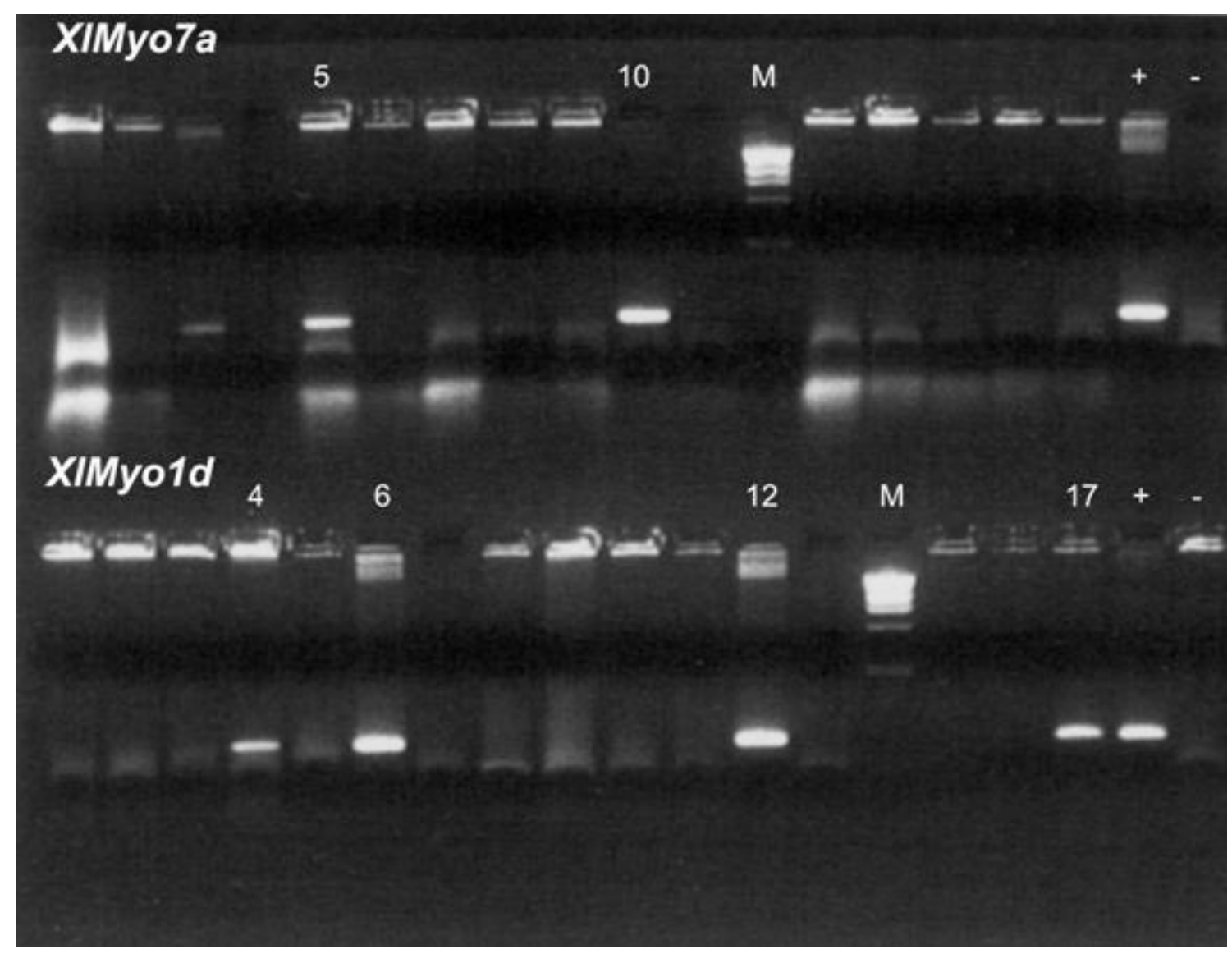

Figure 3. SP screens for two myosin isoforms. SPs from step 3 of Figure 1 were screened in two separate sets of PCR reactions to identify SPs containing 7a cDNA clones (top lanes) or $1 \mathrm{~d}$ (bottom lanes). Using $1 \mu \mathrm{l}$ as a template per PCR, 17 of the 20 SPs were screened. Lanes designated $\mathrm{M}$ contain the same markers as in Figure 2 loaded at a lower concentration. The smallest visible band corresponds to the 506, 517 bp doublet. The 7 a screen identified two positive SPs, numbers 5 and 10, and the $1 \mathrm{~d}$ screen identified four SPs, numbers $4,6,12$, and 17. Lanes designated (+) contain PCR reaction product in which the cloned DNA fragments from which the primer pairs were derived were used as template. Lanes $(-)$ contain products of PCR reactions with no added DNA template. 
which the isoform specific primers were derived and that the rest of the sequence is homologous to the myosin $7 \mathrm{a}$ isoform. (GenBank accession number: DQ100353). Similar results were obtained for the myosin 1d clone (GenBank accession number: AF540952).

\section{DISCUSSION}

The LP method has several distinct advantages and features that make it accessible to researchers on many levels. 1) Screening LPs by PCR eliminates problems with signal-tonoise ratio often encountered with filter hybridization methods. Modifications, such as the use of heterologous or degenerate primers should also be possible. 2) Plate lysates containing live phage can be used directly as template in PCR reactions, eliminating the need for lambda DNA purification. 3) A single lambda clone can be identified in four rounds for a total of 170 PCR reactions (20 SPs; 10 PPs, 40 LPs, $100 \mathrm{pfu}=170$ ). 4) Even with the preparation of PPs, which need to be prepared only once per library, the entire method can be accomplished in under 10 days. If PPs have been prepared for a prior screen, a new clone can be isolated in less than a week. 5) After re-plating positive PPs at low density, LPs can be created in under $1 \mathrm{hr}$ to provide template for the penultimate round of PCR.

The advantages of PCR based screening strategies over traditional filter hybridization based methods, regardless of titre, are many fold. Both approaches require plating of the phage library at high density followed by a plating at low density so the real comparison is at the level of signal detection. First, radioactive probes used to screen libraries must be prepared from cloned fragments or oligo nucleotides of related DNA sequences and modified to contain the radioactive isotope. They have a limited shelf life before having to be prepared again. Although, PCR primers must be designed, synthesized and tested before use, they require no special modification, last indefinitely, and can be used for other applications. Second, a comparison of subsequent steps reveals that the preparation of plaque lifts, hybridization and detection is much more labour intensive than the preparation of plate lysates and direct screening by PCR. The LP method provides an additional benefit of being able to conduct the initial screening for many different cDNA clones without replating the entire library.

The pooling strategy was devised to reduce the number of PCR reactions necessary to identify a positive PP (Figure 1). The number of pfu screened at each round is as follows. In the first round the $20 \mathrm{SPs}$ (a total of $4 \times 10^{5} \mathrm{pfu}$ ) are screened. One SP identifies 10 PPs $\left(2 \times 10^{4} \mathrm{pfu}\right)$ to be screened in the second round. Thus it takes only thirty PCR reactions in two rounds of PCR to identify a positive PP.

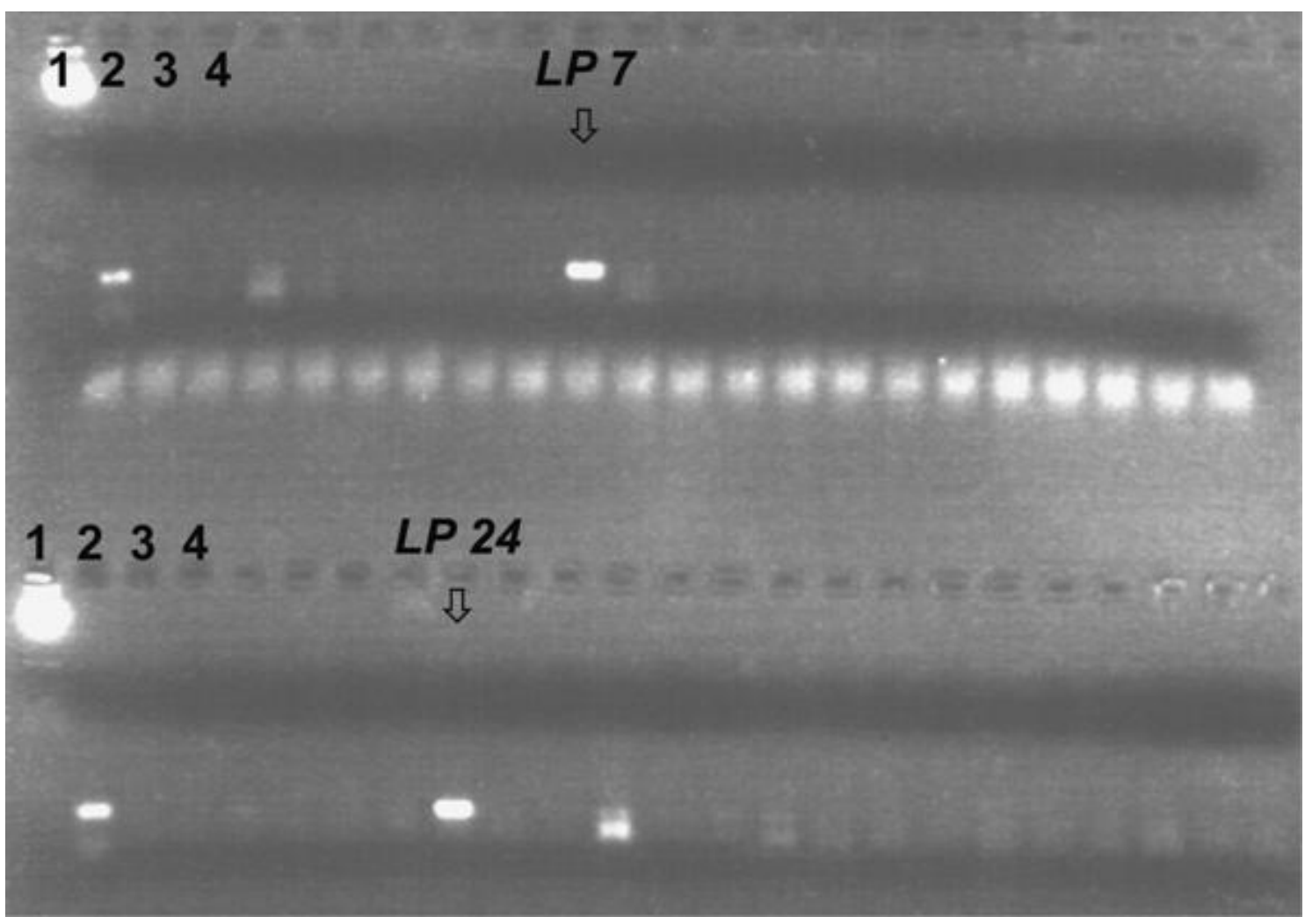

Figure 4. Identification of LPs containing XIMyo7a $\lambda$ gt10 cDNA clones. LPs were prepared as described in Figure 1. Using $3 \mu$ as template in a $20 \mu \mathrm{l} \mathrm{PCR}$ reaction, $40 \mathrm{LPs}$ were screened by PCR using the isoform specific primers for XImyo7a. The products were electrophoresed on a 3\% agarose gel. The 88 bp product was detected in LPs 7 and 24 . On both the top and bottom tiers of the gel, Lambda/HindlII markers were run in lane 1) The following templates were used in the PCR reactions whose products are run in lanes 2-4; lane 2) pXImyo7a, a plasmid containing the fragment from which the isoform specific primers used to screen the library were derived; lane 3) no DNA template; lane 4) APS103, a plate lysate of a purified lambda clone of the XIMyo1d myosin isoform. 
This pool is used to inoculate plates at a density of 100 plaques per plate. The low density plates are used to create 40 LPs that are screened in the third round of PCR (4000 total pfu). Finally, in the fourth round, each of the one hundred plaques (100 pfu) on the positively identified low density plate is screened by PCR. The total number of pfu that are screened to identify a single positive cDNA clone in the four rounds of PCR is 424,100. Alternatively, if time is more critical than reagents, all 200 PPs can be screened simultaneously using a multiwell dish and two thermal cyclers.

As mentioned in the introduction, other PCR based screening strategies that utilize hierarchical pooling diverge when it comes to isolating single clones from the smallest pools used in the first few rounds. Watanabe et al. (1997) use a method to isolate single clones which involves three rounds of replica plating followed by overnight elution of the phage from slices of the original plates and then replating the lysate at sequentially lower density. Although the creation of PPs is initially somewhat more labour intensive, it offers the advantage of being able to re-screen the library very quickly many times.

D'Esposito et al. (1994) report a technique that has been used more recently by Kolbinger et al. (1998) to screen 9.5 $\mathrm{x} 10^{5} \mathrm{pfu}$ using a somewhat similar pooling and re-plating scheme. Although the minimum number of individual PCR reactions is only slightly higher in the D'Esposito et al. 1994 (200 vs 170 for the LP method) two additional platings are required. 192 wells of 32, 6 well plates were used to create top level pools analogous to the PPs described here. The lysates from these top level pools were arrayed in 96 well micro titre dishes and mixed row and column. PCR screening and subsequent re-screening identified a top level pool containing 13,000 pfu which was then plated onto 16 well plates and a similar process described above repeated. The level two pool, containing 450 pfu was again plated onto 24 well plates and rescreened. Finally, 48 single plaque containing pools were plated and a single positive plaque was obtained. The advantage of the LP method is that it utilizes only two platings. In both methods the first plating is done only once, but subsequent platings must be done for each clone that is isolated. In the LP method this is only one additional plating as opposed to three for the D'Esposito method (D'Esposito et al. 1994).

As alternative to screening cDNA libraries to obtain clones, methods such as $5^{\prime}$ and $3^{\prime}$ RACE have been developed (Frohman et al. 1988). PCR amplification to obtain full length cDNAs starting from a small internal sequence requires at least three separate amplifications and several cloning reactions. The $3^{\prime}$ extension using oligo dT is relatively straight forward. A single round of PCR amplification, and cloning into appropriately modified vectors can be accomplished in a few days. However, the $5^{\prime}$ end requires several enzymatic steps, including the 1) randomly primed reverse transcription of mRNA followed by the 2) addition of an adapter via ligation or PCR onto the newly synthesized cDNA. 3) A subsequent round of PCR amplification, using primers complementary to the adapter and cDNA sequence of interest yields the final $5^{\prime}$ end product. Next, the $5^{\prime}$ end cDNA has to be cloned and finally recombined with the $3^{\prime}$ end product to yield a full length transcript. This method is usually efficient for single cDNA isolation. However, when several cDNAs are to be isolated from a single library, the pooling and LP method proves to be a very efficient method, especially if the library is obtained from a colleague or commercial vendor. Once the PPs are made, the library can be re-screened hundreds of times. A single PP can be identified in only two rounds of amplification. Within a minimum of two additional days, a single clone can be identified. Thus this method is highly efficient for isolation of multiple clones from a single library.

Many applications such as drug discovery and structure/function relationship investigations require fulllength cDNA sequences to identify and express the proteins encoded. EST's identified by DNA microarray chip technology are only partial segments of the coding regions of genes. Lambda libraries remain reliable sources of cloned full length, or near full length, cDNAs. The streamlined PCR screening strategy described here improves upon other PCR based methods because the LP technique allows the repeated rapid isolation of specific lambda clones from complex libraries.

\section{REFERENCES}

ALPHEY, Luke. PCR-Based method for isolation of fulllength clones and splice variants from cDNA libraries. BioTechniques, March 1997, vol. 22, no. 3, p. 481-486.

AMARAVADI, Lakshmi and KING, Michael W. A rapid and efficient, non-radioactive method for screening recombinant DNA libraries. BioTechniques, January 1994, vol. 16 , no. 1, p. 98-103.

D'ESPOSITO, Maurizio; MAZZARELLA, Richard; PENGUE, Gina; JONES, Carmela; D'URSO, Michele and SCHLESSINGER, David. PCR-based immortalization and screening of hierarchical pools of cDNAs. Nucleic Acids Research, November 1994, vol. 22, no. 22, p. 4806-4809.

DORFMAN, David M. Amplification of bacteriophage library inserts using polymerase chain reaction. Methods in Enzymology, 1993, vol. 218, p. 336-340.

FROHMAN, Michael A.; DUSH, Michael K. and MARTIN, Gail R. Rapid production of full-length cDNAs from rare transcripts: amplification using a single genespecific oligonucleotide primer. Proceeding of the National Academic of Science of the United States of America, December 1988, vol. 85, no. 23, p. 8998-9002.

GONZALEZ, Daniel H. and CHAN, Raquel L. Screening 
cDNA libraries by PCR using lambda sequencing primers and degenerate oligonucleotides. Trends in Genetics, July 1993, vol. 9, no. 7, p. 231.

KOLBINGER, Frank; STREIFF, Markus B. and KATOPODIS, Andreas G. Cloning of a human UDPgalactose: 2-acetamido-2-deoxy-D-glucose 3betaGalactosyltransferase catalyzing the formation of type 1 chains. The Journal of Biological Chemistry, January 1998, vol. 273 , no. 1, p. 433-440.

McALINDEN, T.P. and KRAWETZ, S.A. A practical method to screen libraries of cloned DNA. Analytical Biochemistry, April 1994, vol. 218, no. 1, p. 237-238.

MUNROE, D.J.; LOEBBERT, R.; BRIC, E.; WHITTON, T.; PRAWITT, D.; VU, D.; BUCKLER, A.; WINTERPACHT, A.; ZABEL, B. and HOUSMAN, D.E. Systematic screening of an arrayed cDNA library by PCR. Proceedings of the National Academy of Sciences of the United States of America, March 1995, vol. 92, no. 6, p. 2209-2213.

REBAGLIATI, M.R.; WEEKS, D.L.; HARVEY, R.P. and MELTON, D.A. Identification and cloning of localized maternal RNAs from Xenopus eggs. Cell, October 1985, vol. 42, no. 3, p. 769-777.

ROSENBERG, Helene F.; CORRETTE, Stephanie E.; TENEN, Daniel G. and ACKERMAN, Steven J. Rapid cDNA library screening using the polymerase chain reaction. BioTechniques, January 1991, vol. 10, no. 1, p. 53-54.

SAMBROOK, Joseph; FRITSCH, Edward F. and MANIATIS, Thomas. Molecular Cloning: A Laboratory Manual. 2nd ed. New York, Cold Spring Harbor Laboratory Press, 1989. 999 p. ISBN 0-87969-309-6.

SOKAC, Anna M. and BEMENT, William M. Regulation and expression of metazoan unconventional myosins. International Review of Cytology, 2000, vol. 200, p. 197304.

TAKUMI, T. Use of PCR for cDNA library screening. Methods in Molecular Biology, 1997, vol. 67, p. 339-344.

WATANABE, Kenji; SAKAI, Fumie and ORII, Hidefumi. Stepwise dilution screening of a cDNA library by polymerase chain reaction. Analytical Biochemistry, October 1997, vol. 252, no. 1, p. 213-214.

YU, Lei and BLOEM, Laura J. Use of polymerase chain reaction to screen phage libraries. Methods in Molecular Biology, 1996, vol. 58, p. 335-339. 